\title{
La base de datos Qualitas Scientiae: proyecto de aplicación del análisis cualitativo de citas a las revistas españolas de biblioteconomía y documentación (1996-2004)
}

\author{
Por José López-Yepes, María-Teresa Fernández-Bajón, Javier Salvador-Bruna \\ y Federico Hernández-Pacheco ${ }^{1}$
}

\begin{abstract}
Resumen: Aplicación del método de análisis cualitativo de citas a las revistas españolas de Biblioteconomía y Documentación en el período 1996-2004 según la muestra empleada por la base de datos IN-RECS iniciada y mantenida por profesores de la Universidad de Granada. El resultado es la base de datos Qualitas Scientiae. Se establece una comparación de las conclusiones de la misma con la base de datos granadina y se aportan nuevos términos derivados de la aplicación.

Palabras clave: Análisis cualitativo de citas, Evaluación de revistas de Biblioteconomía y Documentación, Base de datos Qualitas Scientiae.
\end{abstract}

Title: Qualitas scientiae database: applying qualitative citation analysis to spanish journals of information science (1996-2004)

Abstract: The Qualitas Scientiae database is the result of an application of Qualitative Citation methodology to Spanish Journals in the field of Information Science in the period between 1996 and 2004. The sample of journals follows that of database IN-RECS, developed by the University of Granada. In turn, the findings of Qualitas Scientiae are compared to those of INRECS.

Keywords: Qualitative Citation Analysis, Evaluation of Information Science Journals, Qualitas Scientiae Database.

López-Yepes, José; Fernández-Bajón, María-Teresa; Salvador-Bruna, Javier; Hernández-Pacheco, Federico. "La base de datos Qualitas Scientiae: un proyecto de aplicación del análi-
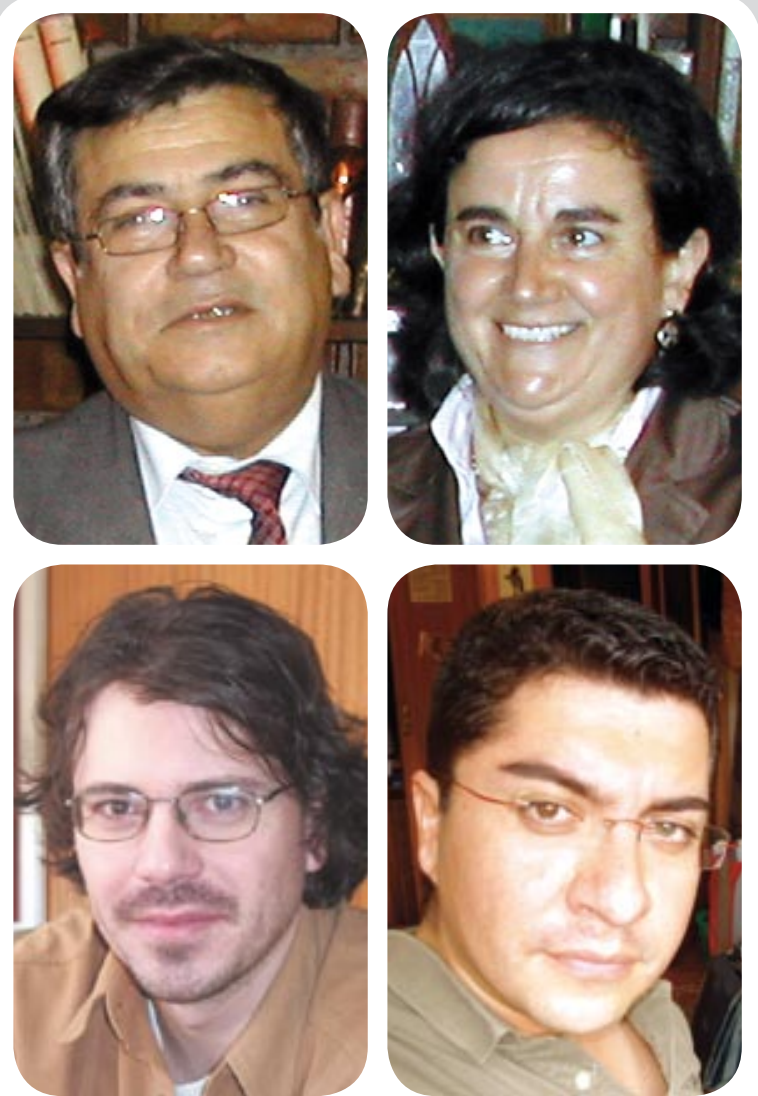

José López-Yepes, María-Teresa Fernández-Bajón, Javier Salvador-Bruna y Federico Hernández-Pacheco sis cualitativo de citas a las revistas españolas de biblioteconomía y documentación (1996-2004)". En: El profesional de la información, 2007, julio-agosto, v. 16, n. 4, pp. 360-367.

DOI: 10.3145/epi.2007.jul.09

Proyecto: Evaluación cualitativa de las publicaciones científicas en humanidades y ciencias sociales (MEC, EA2006-023). La base de datos Qualitas Scientiae (QSci).

LA CULTURA DE LA CALIDAD IMPREGNA TODAS las actividades universitarias y sus consecuencias recaen en la calificación de centros, investigadores, publicaciones, obtención de tramos de investigación, acreditación para la estabilidad del pro- fesorado contratado, influencia en los concursos de habilitación para plazas de funcionarios docentes, adjudicación de contratos subvencionados, etc.

En consecuencia, las aportaciones con ello relacionadas son cada vez más numerosas como se constata en el Observatorio de la calidad en biblioteconomía y documentación mantenido por el departamento complutense. En este contexto, el proyecto $Q S c i$ pretende contribuir a la mejora de los actuales sistemas de evaluación de la producción científica.

http://www.ucm.es/info/multidoc/observatorio/index.htm

http://www.ucm.es/info/multidoc/qualitas/index.htm

Como ya se ha indicado, en nuestros días proliferan los estudios bibliométricos para establecer la calidad de las revistas científicas a partir, esencialmente, de su índice de impacto establecido merced a la presencia de las citas. A conti- 
nuación se muestra una relación de algunos de estos trabajos, descritos más pormenorizadamente en $Q S c i$ $\mathrm{y}$ financiados los cinco primeros por el Ministerio de Educación y Ciencia (Programa de Estudios y Análisis).

- María Dolores Alcaín Partearroyo: Índices del impacto de las revistas españolas de ciencias sociales (biblioteconomía y documentación), psicología, psiquiatría y urbanística y ordenación del territorio) a partir del análisis de las revistas mejor valoradas por los pares, 2003.

\section{http://www.mec.es}

- María Dolores Alcaín Partearroyo: Análisis de las citas dadas por las revistas españolas de derecho y periodismo a partir de las revistas mejor valoradas por los pares. Elaboración de un índice de citas de las revistas españolas de todas las disciplinas de ciencias sociales (Ref. EA2004-00057), 2004.

http://www.mec.es/univ/html/ informes/estudios_analisis/resultados_2004/ea0059/ea0059.pdf

- Adelaida Román Román: Análisis de las citas dadas por las revistas de historia, filología moderna y estudios orientales, árabes y hebráicos, a partir de las revistas mejor valoradas por los pares (Ref. EA2004-0059), 2004.

http://www.mec.es/univ/html/ informes/estudios_analisis/resultados_2004/ea0059/ea0059.pdf

- Adelaida Román; María Dolores Alcaín (investigadores responsables): RESH: Revistas españolas de ciencias sociales y humanas: valoración integrada e índice de citas.

\section{http://www.cindoc.es}

- Evaristo Jiménez Contreras (investigador responsable): IN-RECS. Índice de impacto de las revistas españolas de ciencias sociales (biblioteconomía y documentación, economía, educación, geografía, sociología y psicología). Proyectos EA2003-086, EA20040119, EA2005-0068 y SEJ200408027/SOCI).

http://www.mec.es

http://ec3.ugr.es/in-recs

- Teresa Silió: Bases de datos y sistemas de interrelación de citaciones como indicadores para la evaluación de la ciencia y la tecnología (I Jornadas españolas de indicadores para la evaluación de la ciencia y la tecnología, 2005).

http://www.cindoc.csic.es/info/ fesabid/28.htm

- Félix de Moya Anegón (director). Base de datos de citas de trabajos sobre biblioteconomía y documentación en España (actualizada desde 1983). Granada, Facultad de Biblioteconomía y Documentación. Base de datos construida a partir de las citas recibidas por los artículos referenciados en la base de datos de artículos de revista del Cindoc. No es accesible en internet.

\section{EI problema de la inseguridad evaluativa}

Es evidente que, desde el año 2003 aproximadamente, se ha procedido a realizar estudios encaminados a medir el impacto de revistas y autores españoles en la mayor parte de las áreas de conocimiento. Ello ha supuesto y supone, como justifican los autores de los diversos trabajos, un gran avance en el conocimiento de la valoración y evolución de las distintas disciplinas en un orden eminentemente cuantitativo. Así se hace posible percibir el impacto científico de revistas y autores al poder servirse, entre otros, de indicadores bibliométricos como:

- Índices de impacto de las revistas.

- Instituciones, revistas y artículos más citados.

-Publicaciones que citan a determinadas revistas.
- Citas recibidas por autores y por cada uno de sus trabajos.

- Autocitas.

- Media de citas por documento.

- Vida media de los trabajos de una disciplina a partir de las citas.

- Árboles y redes de citaciones.

De modo más pormenorizado la evidente utilidad de los estudios de esta índole ha sido descrita por los autores de la base de datos $I N$ $R E C S$, la cual "a los gestores, administradores y responsables de agencias de evaluación científica en España les ofrecerá información cuantitativa que les ayudará en sus tareas de evaluación de la investigación publicada. Complementada con valoraciones cualitativas permitirá una mejor evaluación de los científicos y de las instituciones de investigación españolas".

A partir de estas afirmaciones, manifestamos nuestro acuerdo con la clara e imprescindible utilidad de $I N-R E C S$ a los tres primeros destinatarios. Sin embargo, la aplicación (en la medida en que se haga de manera indiscriminada) de los innumerables (valga la redundancia) datos bibliométricos a la evaluación de publicaciones y personas puede ocasionar inseguridad evaluativa y riesgo para "vidas y haciendas" dada la trascendencia que la actividad evaluativa tiene en la promoción del profesorado y de los investigadores. Pero además, la consulta y aplicación de indicadores bibliométricos tampoco asegura, como veremos, una percepción fehaciente del crecimiento, difusión y logros de una disciplina.

Veamos ciertos riesgos derivados de la producción, comportamiento y aplicación de los indicadores obtenidos en los estudios citados y que pueden ocasionar factores de inseguridad evaluativa:

- Algunos de ellos convergen en los mismos objetivos y dan lugar a duplicaciones y a menudo diver- 


\section{"La consulta y aplicación de indicadores bibliométricos tampoco asegura una percepción fehaciente del crecimiento, difusión y logros de una disciplina"}

gencias en los resultados a partir de métodos y fuentes no necesariamente uniformes.

- Los evaluadores podrían ceder a la tentación de aplicar automáticamente determinados indicadores cuando disponen presuntamente de un instrumento considerado válido para ello.

- La selección de revistas-fuente, a pesar de las diversas metodologías contrastadas, no ofrece garantías suficientes a nuestro juicio por el escaso número de las elegidas, la ausencia de artículos de documentación aplicada a determinadas ramas del saber y la limitación cronológica que puede ocasionar la pérdida de una parte de los trabajos de autores todavía activos.

- Las citas ofrecidas por las bases de datos de carácter cuantitativo, como son las que venimos considerando en estas reflexiones, no distinguen entre las que podemos considerar "relevantes" en principio y otras de menor interés como las que contienen datos aclaratorios, de agradecimiento, meras menciones en la bibliografía final, etc. No es, pues y de entrada, suficiente como para asegurar que la mayor acumulación de citas de un artículo es forzosamente sinónimo de calidad y, por consiguiente, no es éste el mejor modo para medir el impacto y la repercusión de la investigación científica.

En estas afirmaciones se basa el origen del proyecto de investigación que estamos presentando como continuación y acrecentamiento de la imprescindible base de datos $I N$ $R E C S$, a cuyos responsables agradecemos la gran ayuda que nos han prestado.

\section{Un nuevo modelo de evaluación de la calidad: el análisis cualitativo de citas}

En la actualidad, como venimos viendo y sobre la base de la experiencia de los países más avanzados científicamente considerados, se está aplicando una serie de indicadores bibliométricos que, como su nombre indica, tratan de calibrar parámetros de carácter cuantitativo como son: número de publicaciones, impacto de las publicaciones periódicas, número de tesis doctorales dirigidas, cantidad de ponencias o comunicaciones presentadas en congresos, etc. El riesgo en dejarse llevar por lo meramente cuantitativo (lo único aparentemente medible) es indudable y lo es más todavía la tendencia a homogeneizar este tipo de evidencias en todas las disciplinas sin establecer las oportunas distinciones. La situación a que nos referimos ha sido tratada con cierta extensión para el área de la biblioteconomía y la documentación, en el trabajo de José López Yepes y Juan Ros García ${ }^{2}$.

Pues bien, a partir de los problemas detectados por los profesores e investigadores de la citada área de conocimientos y que pueden extenderse a los demás, parece conveniente diseñar mediante un proyecto piloto una metodología que, basada en el análisis de citas utilizadas por los investigadores objeto de evaluación $\mathrm{y}$, a su vez recibidas por ellos, permita conocer de modo fehaciente la propagación de sus ideas científicas y la influencia real que han operado en otros investigadores que se han servido de ellas para, teniéndolas como base de conocimiento, obtener otras nuevas ideas. Al fin y al cabo, éste es el procedimiento empleado más habitualmente para hacer posible el crecimiento de los saberes científicos. Y la metodología a la que aludimos trata de resolver los problemas que diversos autores han detectado en relación con la determinación del factor de impacto como instrumento idóneo de la calidad de las revistas y el número de citas como componente de dicho factor y como índice para medir la productividad y calidad de los autores.

En todo caso, el análisis de las citas puede observarse desde dos perspectivas: la cuantitativa, es decir, el número de citas recibidas por un autor, y la cualitativa; esto es, el valor de las citas por su contenido y trascendencia.

Este segundo aspecto va más allá de la mera aplicación del factor de impacto o de su atribución a las obras de los autores. Naturalmente plantea como condición sine qua non la elaboración del índice bibliométrico de citas y la consideración de lo que entendemos como cita de calidad. No lo son, evidentemente, las autocitas, las de agradecimiento, las que contienen meras notas aclaratorias, etc.

La perspectiva cualitativa que supone, como decimos, haber realizado la primera, apenas está esbozada y nosotros hemos contribuido a divulgarla y a manifestar sus ventajas en dos trabajos:

- "Propuesta de método para evaluar trabajos científicos mediante el análisis cualitativo de citas". En: El profesional de la información, 2003, noviembre-diciembre, v. 12, n. 6 , pp. $467-471$.

- "El análisis cualitativo de citas como instrumento para el estudio de la creación y transmisión de las ideas científicas". En: Documentación de las ciencias de la información, 2003, n. 26, pp. 41-70.

Así como en dos trabajos aplicativos del método reseñado: 
- Anuncibay, C., et al. "Análisis cualitativo de citas: la obra de Javier Lasso de la Vega y de Agustín Millares Carlo". En: Documentación de las ciencias de la información, 2005, n. 28, pp. 269-292.

- Salvador Bruna, Javier. "La introducción de las teorías de Otlet en España". En: Documentación de las ciencias de la información, 2006, n. 29, pp. 25-56.

Estamos firmemente convencidos, a tenor de las conclusiones obtenidas en ambos trabajos, que el análisis cualitativo de citas puede ser un método eficaz para conocer en rigor la verdadera aportación de un investigador a su campo de conocimiento y en consecuencia, la auténtica medida de su valor y consiguiente prestigio, aparte de poder evaluar otros elementos como la calidad de las revistas donde publica, el valor de las fuentes que utiliza, etc. Y ello sobre la base de las siguientes razones:

- Es incuestionable que los autores prestigiosos lo son porque las ideas científicas que han obtenido son utilizadas por otros como fundamento de reflexión y fuente de nuevo conocimiento. Si Aristóteles está vigente es porque sus escritos siguen siendo fuente de estudio y reflexión y base para generar nuevos conocimientos.

- La cita que consideramos de calidad lo es porque es portadora de ideas científicas que otros investigadores toman como fuente para obtener las suyas y por ello son utilizadas.

- El estudio de las citas a lo largo de un itinerario cronológico permite conocer cuándo nacen las ideas y cómo se van transmitiendo en el tiempo. Se sabe quiénes son los verdaderos autores de las mismas y en este sentido, se convierten en instrumento útil para percibir el crecimiento y la difusión de un determinado campo del saber.

- También es importante conocer a los autores en los que el investigador evaluado se ha basado para sus trabajos. A ese conjunto de citas lo denominamos "base científica deudora". Complementariamente, la "base científica acreedora" es el conjunto de aquellas en que otros autores recogen las ideas del autor evaluado.

- El método en cuestión consiste (esquemáticamente) en recoger las citas que ha recibido la revista o el autor evaluado, conocer su contenido y las razones de por qué se han formulado y, en último término, reunir también las ideas o juicios aportados por el autor evaluado a través de las citas que aporta.

- La aplicación del método a las revistas permitirá obtener su factor de impacto a partir de las citas de calidad y no del conjunto de ellas como ahora se efectúa de modo indiscriminado. El resultado será el índice de impacto cualitativo. Aplicado a las citas recibidas por los autores permitirá conocer el auténtico impacto de su valor y aportación a su campo del saber.

- Asimismo, la utilización del método de evaluación mediante citas de calidad permitirá conocer otros factores como: a) la base científica deudora de una revista o un autor al determinar qué fuentes ha utilizado y su valor; b) la base científica acreedora de una revista o de un autor al conocer dónde han

\footnotetext{
"Los autores prestigiosos lo son porque las ideas que han obtenido son utilizadas por otros como fundamento de reflexión y fuente de nuevo conocimiento"
}

sido citados; c) la relación y temática de las ideas científicas concebidas y transmitidas a lo largo de un período así como de los autores de las mismas como contribución a la historia de la investigación de una determinada disciplina; d) la visibilidad nacional e internacional de revistas y autores; e) cómo se ha producido la formación de focos y frentes de investigación y la formación de escuelas científicas.

\section{Objetivos del proyecto QSci}

El proyecto que ahora presentamos se basa en el interés general para el sistema universitario español y persigue los siguientes objetivos:

- Definir un nuevo método aplicable a la evaluación más precisa y rigurosa de la calidad de las revistas científicas mediante la elaboración del factor de impacto sobre la base de las citas de calidad y aplicable, asimismo, a la evaluación de las obras de los autores.

- Diseñar una herramienta informática que facilite la aplicación del nuevo método y pueda obtener los objetivos enumerados en el punto anterior.

- Establecer un modelo a partir de la aplicación del nuevo método a una muestra de revistas científicas españolas y autores representativos de todos los campos del saber: humanidades, ciencias sociales y jurídicas, ciencias experimentales, enseñanzas técnicas y ciencias de la salud en un período de tiempo determinado. Los resultados se han cifrado en la producción de los siguientes indicadores:

a. Autores:

- Listado de citantes.

- Listado de citados.

- Citantes según temática.

- Citados según temática.

b. Base intelectual:

- Deudora. 
- Acreedora.

c. Revistas:

- Citantes.

- Citadas.

- Citantes según temática.

- Citadas según temática.

- Factor de impacto cuantitativo y cualitativo.

\section{d. Artículos:}

- Citantes.

- Citados.

e. Instituciones:

- Citantes.

- Citadas.

- Citantes según temática.

- Citadas según temática.

f. Ideas científicas: gía.

- Citas totales según su tipolo-

- Ideas científicas de los autores citantes y de los autores citados.

- Textos de las ideas científicas citadas.

- Ideas científicas de las revistas citantes y de las revistas citadas.

- Ideas científicas de las instituciones citantes y citadas.

http://www.ucm.es/info/qualitas/biblio/biblio.htm

\section{Conclusiones}

- La base de datos $Q S c i$ recoge el tratamiento de datos de acuerdo con la metodología del análisis cualitativo de citas y los resultados consiguientes.

- Los responsables del proyecto parten del hecho considerado cierto de que los análisis cuantitativos de citas como los llevados a cabo por las bases de datos españolas IN$R E C S$ y $R E S H$ y la norteamericana Citation Index pueden no constituir una herramienta íntegramente vá- lida para la evaluación de autores, trabajos, revistas e instituciones por cuanto, al menos un $50 \%$ de las citas no son de calidad, es decir, no son transmisoras de ideas científicas y por tanto no significan soporte para la obtención de nuevas ideas por parte de los autores citantes y correlativamente repercutirán en la medida del prestigio de los autores citados.

- La muestra utilizada en el proyecto corresponde a un elevado porcentaje (unas 500 citas) de las examinadas por los autores de la base de datos IN-RECS (excluidas las procedentes de artículos de revistas fuera del área de biblioteconomía y documentación). Todas nos han sido facilitadas por los responsables de aquélla y a los que, desde estas líneas, expresamos nuestro más sincero agradecimiento.

- Procede indicar que las citas adoptadas por IN-RECS proceden del ámbito de revistas publicadas en el período 1994-2004 y que se excluyen citas procedentes de monografías, de revistas extranjeras así como de actas de congresos y otras reuniones científicas.

- Las novedades que pretende aportar el proyecto $Q S c i$ parten de la aplicación del denominado análisis cualitativo de citas y son, fundamentalmente y entre otras, las siguientes:

a. Introducción, si así lo aceptara nuestra comunidad científica, de nuevos conceptos (véase glosario) expresados en los siguientes términos: análisis cualitativo de citas, base intelectual deudora y acreedora, cita de calidad, cita de idea, cita de contexto, cita en la bibliografía final, indicadores de calidad, inseguridad evaluativa y método del análisis de citas.

b. Reconocimiento para aquellos autores, artículos, revistas e instituciones que han recibido más citas de calidad y, por tanto, revisten mayor mérito. c. Examen de los autores, artículos, revistas e instituciones citantes y sus preferencias por determinados autores, relación que significa una base para el ulterior establecimiento de escuelas científicas.

d. Posibilidad de determinar con mayor precisión temática las líneas de investigación creadas y participadas entre y por autores, artículos, revistas e instituciones citantes y citados.

e. Posibilidad de conocer los itinerarios seguidos por las ideas contenidas en las citas.

f. Posibilidad de conocer con cierta precisión las ideas científicas creadas por los autores (base intelectual acreedora) y su uso por los autores citantes (base intelectual deudora).

Veamos estas aportaciones de un modo más pormenorizado (consultar este url para obtener los datos propiamente dichos):

http://www.ucm.es/info/multidoc/qualitas/biblio/biblio.htm

\section{Autores}

- De los citantes se proporciona el listado de los 25 con las citas de calidad utilizadas, el número de medio de citas por trabajo y el porcentaje de citas de calidad en relación con autores y trabajos.

- De los citados, se ofrece la lista de los que lo son más veces tanto en citas de calidad como de no calidad y el término medio de autores que han recibido citas de calidad.

- Finalmente, es también objeto de descripción la temática (descriptores temáticos) de los trabajos publicados por los autores citantes y citados.

\section{Base intelectual deudora $y$} acreedora

- La primera sirve para conocer a los autores y trabajos que constituyen la base que permite a 


\section{Glosario de término relacionados con el proyecto}

\section{- Análisis cualitativo de citas}

Operación y resultado de detectar el número de citas de calidad recibidas por autores, artículos, instituciones y revistas y deducir de ello determinados indicadores como el factor - índice de calidad así como la posibilidad de que el estudio de dichas citas de calidad permita seguir el itinerario o hilo conductor de las ideas científicas desde su creador hasta los siguientes autores que las han utilizado como base de reflexión para la obtención de sus ideas científicas propias.

\section{- Análisis cuantitativo de citas}

Operación y resultado de detectar el número de citas recibidas por autores, artículos, instituciones y revistas y deducir de ello determinados indicadores como el factor o índice de impacto como expresión de calidad.

\section{- Base científica acreedora de un autor} Conjunto de ideas científicas de un autor expresadas en citas de sus trabajos utilizados por otro autor.

\section{- Base científica deudora de un autor} Conjunto de ideas científicas contenidas en citas utilizadas por un autor para obtener los resultados de su investigación gracias en buena parte al concurso de los autores citados.

\section{- Cita de calidad}

Aquélla que es portadora de una idea científica utilizada como base por los autores citantes para la obtención de las suyas e instrumento que permite describir el itinerario de las ideas científicas desde su creación. No son citas de calidad a efectos de evaluación las autocitas, las que tienen una finalidad aclaratoria, de agradecimiento, las situadas tan sólo en la bibliografía final de un trabajo, etc.

\section{- Cita de contexto}

Aquella cita de calidad que presenta panoramas de ideas o corrientes de pensamientos expresadas por dos o más autores.

\section{- Cita de idea}

Aquella cita de calidad que es portadora de una idea científica claramente presentada. Se considera cita de calidad pero más valiosa que la de contexto.

\section{- Indicadores de calidad}

Aquellos obtenidos mediante el examen de las citas de calidad.

\section{- Inseguridad evaluativa}

Sensación que afecta al sujeto evaluado y conducta susceptible de ser mostrada por los evaluadores en la medida en que los dispositivos y herramientas de evaluación son todavía manifiestamente mejorables desde las siguientes consideraciones: a) los estudios y bases de datos de evaluación mediante el recuento de citas convergen en los mismos objetivos pero no siempre proporcionan resultados similares u homogéneos; b) cabe el riesgo de utilizar herramientas de evaluación de modo mecánico a partir de rankings previstos en las citadas herramientas; c) la selección de revistas-fuente puede ofrecer aspectos dudosos así como la discriminación de determinados tipos de documentos, períodos cronológicos, nacionalidad de los autores, etc; d) las citas ofrecidas por las bases de datos cuantitativas no distinguen la relevancia de las mismas $y$, en consecuencia, su mero recuento no es forzosamente sinónimo de calidad o impacto.

- Método del análisis cualitativo de citas Método de evaluación científica y de estudio de evolución de las ideas científicas basado en el examen de las citas de calidad presentes en un texto científico. Ha sido puesto en práctica por algunos investigadores de la evaluación científica.

\section{Reducción del embargo online de EPI}

EPI ha bajando ya a 1 año (6 números) el tiempo de carencia o de embargo (time embargo, time wall) de acceso de pago a la versión online de la revista.

En consecuencia se han liberado los contenidos del periodo enero 2000-julio 2006, que están en acceso abierto en la web de la propia revista:

http://www.elprofesionaldelainformacion.com

El periodo 1992-1999 está disponible en html y el 2000-2006 en pdf. 
un autor obtener nuevas ideas científicas, lo que hace posible conocer el valor de sus "deudores", las temáticas inspiradas en los mismos, las líneas de investigación y la posible existencia de lazos de comunicación o escuelas científicas. Ello responde a la siguiente expresión: un autor publica sobre cita a

- La base intelectual acreedora sirve para conocer qué autores y qué ideas creadas por los mismos han sido aprovechadas por otros para hacer crecer el campo de su línea de investigación. Ello respondería a la siguiente expresión: un autor es citado por en las materias de

- La base de datos QSci ofrece información correspondiente a las bases deudora y acreedora, en los términos arriba señalados, de 45 autores deudores y 44 autores acreedores.

\section{Revistas}

- Nuestra base de datos ofrece el total de citas contenidas en las revistas-fuente, distinguiendo el porcentaje de citas de calidad.

- Asimismo ofrecemos la relación de las revistas citadas a lo largo de la base de datos estableciendo una jerarquía en función de la tipología de las citas.

- Damos a conocer, además, la temática de los artículos publicados.

- Finalmente, determinamos el factor de impacto de las diferentes revistas estableciendo la correspondiente diferenciación entre el factor de impacto obtenido en IN-RECS (FI IN), el factor de impacto cuantitativo de QSci (FI) y el factor de impacto cualitativo de QSci (FIC), bien distinto. La diferencia entre FI IN y FI se debe a que nuestra base no ha tratado, como se ha indicado al principio, todos los artículos citados en IN-RECS.

\section{Artículos}

- Las dos primeras tablas ofrecen los artículos con mayor número citas y citas de calidad empleadas.

- La tabla siguiente presenta los 25 artículos más citados incluyendo todo tipo de citas.

- La última tabla presenta los diez artículos que han recibido más citas de calidad.

\section{Instituciones}

Son las organizaciones profesionales, docentes y de investigación, en último término, las responsables del quehacer de investigación y de estudio que da lugar a los trabajos científicos. Son, pues, también objeto de evaluación y por consiguiente, de obtener prestigio. En este sentido QSci ofrece los siguientes datos:

- Las instituciones proveedoras de trabajos con más citas emitidas y según el número de citas de calidad.

- Las más citadas y su clasificación según citas de calidad recibidas.

- Las citantes según la temática estudiada.

- Las citadas según la temática estudiada.

\section{Ideas científicas}

Las tablas contenidas en este punto constituyen una herramienta de gran repercusión a la hora de ejercer actividades de evaluación científica.

Leer EPI es como asistir a un curso de formación continua pero con el horario acomodado a tus necesidades.
- De los más de 500 artículos examinados ofrecemos las cifras totales de citas recibidas según su tipología, de lo que resulta ser de calidad tan sólo un 50\% aproximadamente. Este es un dato que, en nuestra opinión, resta valor al mero recuento de citas.

- Paralelamente, se ofrece la distribución de las citas y sus tipos durante el período 1996-2004 y la tasa media de citas por artículo citante y citado según tipología de las citas.

- La transmisión de las ideas científicas y su contenido textual se ofrece en las tablas dedicadas a las ideas científicas de los autores citados conjuntamente con los autores citantes.

\section{Relación IN-RECS/Qualitas Scientiae}

Como se ha expresado anteriormente, la experiencia de $Q S c i$ se basa en un elevado porcentaje de los artículos y sus citas empleados por IN-RECS; en concreto no se han tenido en cuenta las citas procedentes de revistas ajenas al campo de la biblioteconomía y la documentación. Ello ha motivado que, en la comparación relativa al factor de impacto de las revistas, nosotros distingamos entre FI IN (índice de impacto de IN-RECS) y nuestros FI (factor de impacto de citas globales) y FI C (factor de impacto cualitativo).

$I N-R E C S$ proporciona al día de hoy tres grupos de indicadores:

- El índice de impacto cuantitativo de las revistas de biblioteconomía y documentación a partir de seis revistas-fuente a lo largo del período 1996-2004 por años y acumulativamente.

- El número de citas recibidas por los trabajos y autores considerados en las revistas-fuente.

- La relación de los 100 artículos de revista más citados en el 
período y sobre la base del apartado anterior.

A partir de estos presupuestos, creemos que es útil y oportuno establecer una comparación, aun demasiado aleatoria que se pueda considerar dada la cortedad de la muestra en el contexto de todas las revistas, entre IN-RECS y $Q S c i$ en los apartados en que ello es posible:

a. Los factores de impacto cuantitativo y cualitativo proporcionados por ambas bases de datos se observan en las tablas $2.7,2.8$ y 2.9 (ver url del proyecto) para las revistasfuente, esto es, El profesional de la información, Item, Revista general de información y documentación, Boletín de la Anabad, Revista española de documentación científica y Cybermetrics.

b. La tabla 1 muestra los nueve autores más citados según el análisis de $I N-R E C S$ y el de $Q S C i$ distinguiendo todas las citas obtenidas en nuestra muestra (un pequeño porcentaje inferior) señalada por la expresión QSCi global y las citas de calidad bajo la expresión QSCi C.

En suma la lista de los nueve autores que han recibido más citas de calidad queda como se observa en la tabla 2.

c. En la lista de los cien artículos más citados ofrecida por IN-RECS sólo tenemos en cuenta las citas procedentes de las revistas nacionales y establecemos la comparación con los datos ofrecidos por QSci. Como puede observarse, la coincidencia del número de citas de la base de la Universidad de Granada con la nuestra es casi absoluta. Sin embargo, de los 44 artículos que registran al menos 3 citas nacionales en IN-RECS solamente los diez mostrados en la tabla 2 contienen 3 citas de calidad, es decir, menos del 25 por ciento.

http://www.ucm.es/info/multidoc/qualitas/index.htm

\section{Conclusión final}

El modelo obtenido en el presente trabajo y expuesto a través de la base de datos QSCi se basa en el método del análisis cualitativo de citas con dos propósitos: servir de instrumento de evaluación de autores, trabajos, revistas e instituciones y conocer la creación y evolución de las ideas científicas en todo campo del saber por medio del examen de la base intelectual de autores citantes y citados. La aplicación práctica del primer propósito debe llevarse a cabo como complemento de los indicadores cuantitativos provistos por la base de datos IN-RECS y no como herramienta exclusiva de evaluación. En

\begin{tabular}{|l|c|c|}
\hline \multicolumn{1}{|c|}{ Autor } & Orden & QSci \\
\hline Moya-Anegón & 1 & 13 \\
\hline Codina & 2 & 9 \\
\hline Frías & - & 9 \\
\hline Moscoso & 4 & 7 \\
\hline Barrueco & - & 7 \\
\hline García-Testal & 6 & 6 \\
\hline Moreiro & 7 & 5 \\
\hline López-López (*) & - & 5 \\
\hline $\begin{array}{l}|c| \\
\text { Ortiz-Repiso }\end{array}$ & 9 & 2 \\
\hline $\begin{array}{l}\text { (*) Hay citas recibidas por este autor procedentes } \\
\text { de revistas ajenas al área que no han sido exami- } \\
\text { nadas por QSci. Por tanto, el número de citas de } \\
\text { calidad aparece previsiblemente incompleto }\end{array}$ \\
\hline
\end{tabular}

Tabla 2

todo caso, nuestro proyecto debería culminar su éxito en ambos propósitos analizando todos los trabajos aparecidos en forma de artículos, monografías y actas de congresos del área de la biblioteconomía y la documentación en un período de tiempo más dilatado.

\section{Notas}

1. Grupo de Investigación Otlet, Departamento de Biblioteconomía y Documentación de la Universidad Complutense de Madrid.

2. López Yepes, José; Ros García, Juan. "Problemas relativos a la evaluación científica de los profesores del área de biblioteconomía y documentación". En: Revista general de información y documentación, 2003, v. 13, n. 2, pp. 37-58.

José López Yepes, catedrático, Facultad de Ciencias de la Información, UCM.

yepes@ccinf.ucm.es

María Teresa Fernández Bajón, profesora titular, Facultad de Ciencias de la Documentación, UCM. maite@caelo.eubd.ucm.es

Javier Salvador Bruna, técnico, Cindoc-CSIC, Madrid.

salvador@cindoc.csic.es

Federico Hernández Pacheco, profesor ayudante, Facultad de Ciencias de la Información, UCM. profhern@yahoo.com 